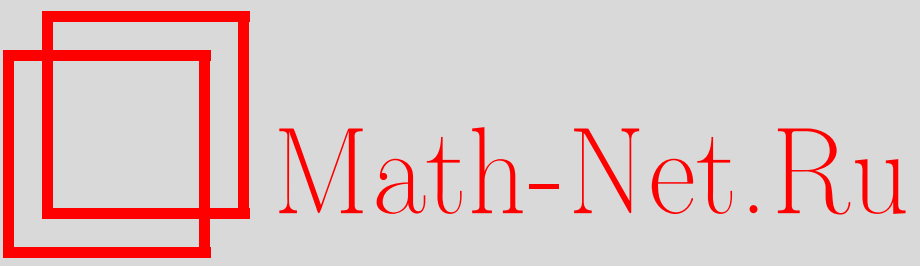

Н. Г. Плетнёв, Д. В. Серебрякова, Супералгебра конформных деформаций твистороподобных суперпространств, ТМФ, 1997, том 110, номер 1, 114-121

DOI: https://doi.org/10.4213/tmf956

Использование Общероссийского математического портала Math-Net.Ru подразумевает, что вы прочитали и согласны с пользовательским соглашением

http://www . mathnet.ru/rus/agreement

Параметры загрузки:

IP: 18.234 .156 .22

26 апреля 2023 г., $07: 25: 15$ 
ТЕОРЕТИЧЕСКАЯ

И МАТЕМАТИЧЕСКАЯ

ФИЗИКА

Том 110, № 1

январь, 1997

Н.Г. Плетнев* , Д. В. Серебрякова*

\title{
СУПЕРАЛГЕБРА КОНФОРМНЫХ ДЕФОРМАЦИЙ ТВИСТОРОПОДОБНЫХ СУПЕРПРОСТРАНСТВ
}

\begin{abstract}
Описано твистороподобное расширение $N=2$ конформного суперпространства с нетривиальными центральными зарядами. Параметры новых (анти)коммутирующих генераторов - носителей индекса спиновой $S U(2)$-симметрии используются для построения безмассовых гармоник, позволяюших резко уменьшить число существенных координат. Обычные векторные координаты при таком подходе становятся составными, а число грассмановых сокращается до одной, причем скалярной относительно преобразований Лоренца. Построенная замкнутая алгебра генераторов позволяет ковариантно редуцировать теорию из исходного суперпространства, где затруднен выбор подходящих аналитических подпространств, на суперпространство существенно меньшей грассмановой размерности. Специальное определение координат и плоского пространства для этой структуры позволяет естественно получить твисторное уравнение Пенроуза, а также гармоники Огиевецкого.
\end{abstract}

\section{1. ВВЕДЕНИЕ}

Разработка единого подхода к квантовой физике и геометрии пространства-времени, основанного на использовании комплексных структур, породила интерес к изучению геометрии специальной теории относительности в свете твисторных объектов.

Основной результат твисторного подхода состоит в том, что различные модели квантовой теории поля могут определяться не столько уравнениями движения (или принципом действия), сколько внутренними свойствами комплексных проективных многообразий $(\mathrm{CP})$, находящихся в твисторном соответствии с комплексифицированным пространством Минковского (СМ). Тем самым взаимодействие определяется комплексной структурой СР. Характерным свойством этой конструкшии является свойство расшепления 1-функций на in- и out-состояния. Конформная инвариантность в СМ в действительности вторична по отношению к голоморфности и свойствам расщепления.

Проблема интерпретации гравитации и теорий с нарушенной конформной инвариантностью в твисторных понятиях представляется как описание подходящих деформаций плоского СР (3). Число используемых плоских твисторных пространств довольно велико, но для описания массивных частиц со спином, потенциалов внутренних симметрий, уравнений с источниками необходимы приспособленные для этих задач обобшения твисторных конструкций. Так, переф̆ормулировки действия для суперчастиц и суперструн

${ }^{*}$ Институт математики СО РАН 
(на языке твисторов) [1] прояснили происхождение $k$-симметрии Зигеля и позволили последовательно провести процедуру ковариантного квантования. В существующих подходах без твисторизации связи не только выделяют мультиплет, но и определяют свободные уравнения движения, что затрудняет включение взаимодействия, анализ спонтанного нарушения симметрии, процедуру квантования.

Концепция гармонического $N=2$ суперпространства [2], развитая для решения такого рода проблем, напоминает твисторньй подход, в котором свободные поля на "своем" (супер)пространстве порождают физические (супер)поля, подчиняющиеся (связям) уравнениям движения. Однако пути, ведущие от уравнений (связей) к твисторам, основаны больше на догадках, чем на конструктивной основе.

Наша цель - дать последовательное описание твистороподобного расширения $N=2$ конформного суперпространства с нетривиальными центральными зарядами.

\section{2. ОБОЗНАЧЕНИЯ И ОБОСНОВАНИЯ}

В этом разделе мы представляем генераторы, образующие предлагаемую суперконформную алгебру, и перечисляем отправные требования, которыми руководствовались при получении этой структуры. Мы пользуемся стандартными обозначениями и соглашениями двухкомпонентного спинорного формализма [3].

В основу нашей конструкции положен стандартный набор генераторов, отвечающих конформной группе Пуанкаре [3]: $P_{\alpha \dot{\alpha}}$ - генератор сдвига, $K_{\alpha \dot{\alpha}}$ - генератор конформных преобразований, $L_{\alpha \beta}, L_{\dot{\alpha} \dot{\beta}}-$ лоренцевы повороты и $D$ - дилатация. Здесь $\alpha$ и $\dot{\alpha}$ - спинорные индексы представлений $\left(\frac{1}{2}, 0\right)$ и $\left(0, \frac{1}{2}\right)$ группы Лоренца. Действие генераторов $L_{\alpha \beta}\left(L_{\dot{\alpha} \dot{\beta}}\right)$ на любой оператор $\widehat{M}_{\gamma}$ - носитель такого же индекса - нормировано стандартным образом:

$$
\left[\widehat{L}_{\alpha \beta} \widehat{M}_{\gamma}\right]=\varepsilon_{\gamma(\alpha} \widehat{M}_{\beta)} .
$$

Желание рассматривать исходные векторные координаты $x^{\alpha \dot{\alpha}}$ из СМ как вторичные величины - требование, обусловливающее инцидентность твистора $Z^{a}$ точке $x \in$ $\mathrm{CM}[4],-$ привело к идее своеобразного извлечения квадратного корня из конформной суперсимметрии. Эта процедура реализована введением непаулевских твистороподобных генераторов $\left(q_{\alpha i}^{+}, p_{\dot{\alpha} i}^{-}\right)$и обратных им по отношению к $D$-градуировке $\left(s_{\alpha i}^{-}, k_{\dot{\alpha} i}^{+}\right)$, таких что

$$
\left[q_{\alpha i}, p_{\dot{\alpha} j}\right] \sim \varepsilon_{i j} P_{\alpha \dot{\alpha}}, \quad\left[s_{\alpha i}, k_{\dot{\alpha} j}\right] \sim \varepsilon_{i j} K_{\alpha \dot{\alpha}} .
$$

При этом мы полагаем операторы $\hat{q}_{\alpha i}, \hat{s}_{\alpha i}$ безразмерными по $D$, для того чтобы параметры $x^{\alpha i}$ при $\hat{q}_{\alpha i}$ и $\omega^{\alpha i}$ при $\hat{s}_{\alpha i}$, будучи также безразмерными, могли служить параметрами бесконечного разложения (супер)поля $\Phi\left(x_{\alpha i}\right)$. Здесь $( \pm)$ - фиксация веса по отношению к новому заряду $A$, имеюшему смысл дилатации в пространстве твистороподобных параметров. Индекс спиновой $S U(2)$-симметрии преобразуется генератором $J_{i j}$, который мы нормировали следуюшим образом:

$$
\left[J_{i j}, q_{\alpha k}\right]=\frac{1}{2} \varepsilon_{k(i} q_{\alpha j)}, \quad\left[J_{i j}, J_{k l}\right]=\varepsilon_{i(k} J_{l) j} .
$$

Введение этой симметрии позволит нам в дальнейшем получить объекты, непосредственно связанные с гармониками Огиевецкого [5]. 
Уже на этом этапе конструкция получилась замкнутой и может быть расширена в двух направлениях.

1. Привлечение антикоммутирующих генераторов суперсдвигов $Q_{\alpha I}, Q_{\dot{\alpha} I}$ и суперконформных бустов $R_{\alpha I}, R_{\dot{\alpha} I}$. В настоящей работе рассматривается случай изотопического индекса для $N=2$, преобразуюшегося по представлению группы $S U(2)$ :

$$
\left[B_{I J}, Q_{\alpha K}\right]=\frac{1}{2} \varepsilon_{K(I} Q_{\alpha J)} .
$$

Введение $Q_{\alpha I}, Q_{\dot{\alpha} I}$, "порождаюших" сдвиг $P_{\alpha \dot{\alpha}}:\left\{Q_{\alpha I}, Q_{\dot{\alpha} J}\right\}=-\varepsilon_{I J} P_{\alpha \dot{\alpha}}$, естественным образом влечет за собой появление нового заряда $B$ грассмановой киральности

$$
\left[B, Q_{\dot{\alpha} I}\right]=\frac{1}{2} Q_{\alpha I} .
$$

Требование замкнутости приводит к возникновению новых антикоммутирующих генераторов $Q_{i I}, R_{i I}$, которые, являясь скалярами по отношению к группе Лоренца, играют важнейшую роль во внутренней организации нашей конструкции твистороподобного расширения (супер)конформной группы Пуанкаре. На этом этапе алгебра является замкнутой, причем не только для $N=2$, но и для $N=1$.

2. Вторым направлением развития расширенной твисторами конформнойгруппы Пуанкаре является согласование ее с алгеброй, описывающей пространство с нетривиальными центральными зарядами и лоренц-подобными преобразованиями, перемешивающими новые не калуца-клейновские размерности с пространственными. Это стремление обусловлено тем, что давно известный путь [6] преодоления "по-gо"-барьера для вспомогательных полей суперсимметричных теорий состоит в рассмотрении суперпространства с центральными зарядами, которые могут возникать как дополнительные импульсы при размерной редукции из $D=10,11$.

Замкнутая алгебра включает генераторы центральных зарядов $Z, \bar{Z}$ (соответственно $Y, \bar{Y}$ в конформном секторе), а также подалгебру $\left(W_{\alpha \dot{\alpha}}, \bar{W}_{\alpha \dot{\alpha}}, L_{\dot{\alpha} \dot{\beta}}, L_{\alpha \beta}, B\right)$, изоморфную $S O(1.5)[6]$. Таким образом, задача состоит в расширении супералгебры с центральными зарядами путем введения спиновой $S U(2)$-симметрии, для того чтобы сузить набор сушественных координат за счет привлечения безмассовых гармоник, которые, в свою очередь, конструируются из параметров, соответствуюших генераторам полученной группы. Такое расширение сушествует, но жесткость условий, накладываемых тождествами Якоби, требует введения новых коммутирующих спиноров $\left(\omega_{\dot{\alpha} i}, \bar{\omega}_{\dot{\alpha} i}, Z_{\alpha i}, Y_{\alpha i}\right)$ и дополнительных центральных зарядов $\widetilde{Z}, \widetilde{Y}$ :

$$
\begin{array}{lll}
{\left[Y, q_{\alpha i}\right] \sim Y_{\alpha i},} & {\left[\bar{Y}, p_{\dot{\alpha} i}\right] \sim \omega_{\dot{\alpha} i},} & {\left[\widetilde{Y}, p_{\dot{\alpha} i}\right] \sim \bar{\omega}_{\dot{\alpha} i},} \\
{\left[Z, s_{\alpha i}\right] \sim Z_{\alpha i},} & {\left[\bar{Z}, k_{\dot{\alpha} i}\right] \sim \bar{\omega}_{\dot{\alpha} i},} & {\left[\widetilde{Z}, k_{\dot{\alpha} i}\right] \sim \omega_{\alpha i} .}
\end{array}
$$

Само по себе это расширение тоже является замкнутой алгеброй. Более того, согласование этой алгебры с суперконформной группой, представленной в п.1, не требует привлечения дополнительных генераторов, а только устанавливает ряд нетривиальных коммутационных соотношений между генераторами этих подгрупп.

Таким образом, супералгебра - предмет настояшей статьи - состоит из 31 генератора: 4 векторных $\left(P_{\alpha \dot{\alpha}}, K_{\alpha \dot{\alpha}} ; W_{\alpha \dot{\alpha}}, \bar{W}_{\alpha \dot{\alpha}}\right), 8$ твисторных $\left(q_{\alpha i}, s_{\alpha i} ; p_{\dot{\alpha} i}, k_{\dot{\alpha} i} ; \omega_{\dot{\alpha} i}, \bar{\omega}_{\dot{\alpha} i} ;\right.$ 
$\left.Z_{\alpha i}, Y_{\alpha i}\right), 6$ центральных зарядов $(Z, Y ; \bar{Z}, \bar{Y} ; \widetilde{Z}, \widetilde{Y}), 6$ антикоммутируюших генераторов из суперсектора $\left(Q_{\alpha I}, R_{\alpha I} ; Q_{\dot{\alpha} I}, R_{\dot{\alpha} I} ; Q_{i I}, R_{i I}\right), 3$ генераторов градуировки $(D, A, B)$, генераторов четырех $S U(2)$-врашений $\left(L_{\alpha, \beta}, L_{\dot{\alpha}, \dot{\beta}}, J_{i j}, B_{I J}\right)$. Мы объединяем эти генераторы с соответствуюшими генераторами из конформного сектора в пары, поскольку гармонические структуры возникают при определении плоских пространств именно на парах типа $\left(x_{\alpha i}, \omega_{\alpha i}\right)$.

\section{3. ОПИСАНИЕ СУПЕРАЛГЕБРЫ}

В этом разделе приведен полный набор коммутаторов, который почти однозначно (с произволом относительно нескольких численных коэффициентов) фиксируется тождествами Якоби.

Градуировка. Сначала приведем градуировку всех генераторов описываемой алгебры. Для наглядности сопоставим каждому генератору строку $(\delta, \alpha, \beta)$, где числа $\delta, \alpha$ и $\beta$ определяются для любого генератора $\widehat{M}$ как $[D, \widehat{M}]=\delta \widehat{M},[A, \widehat{M}]=\alpha \widehat{M}$, $[B, \widehat{M}]=\beta \widehat{M}$, тогда имеем

$$
\begin{aligned}
& Q_{\alpha I} \leftrightarrow\left(-\frac{i}{2}, 0, \frac{1}{2}\right) \leftrightarrow-R_{\alpha I}, \quad Q_{\dot{\alpha} I} \leftrightarrow\left(-\frac{i}{2}, 0,-\frac{1}{2}\right) \leftrightarrow-R_{\dot{\alpha} i}, \\
& Q_{i I} \leftrightarrow\left(-\frac{i}{2},-i, \frac{1}{2}\right) \leftrightarrow-R_{i I}, \\
& Z_{\alpha i} \leftrightarrow(-i,-i, 1) \leftrightarrow-Y_{\alpha i}, \quad p_{\dot{\alpha} i} \leftrightarrow(-i,-i, 0) \leftrightarrow-k_{\dot{\alpha} i}, \quad P_{\alpha \dot{\alpha}} \leftrightarrow(-i, 0,0) \leftrightarrow-K_{\alpha \dot{\alpha}}, \\
& q_{\alpha i} \leftrightarrow(0, i, 0) \leftrightarrow-s_{\alpha i}, \quad \bar{\omega}_{\dot{\alpha} i} \leftrightarrow(0, i,-1) \leftrightarrow-\omega_{\dot{\alpha} i}, \quad W_{\alpha \dot{\alpha}} \leftrightarrow(0,0,1) \leftrightarrow-\bar{W}_{\alpha \dot{\alpha}}, \\
& Z \leftrightarrow(-i, 0,1) \leftrightarrow-Y, \quad \bar{Z} \leftrightarrow(-i, 0,-1) \leftrightarrow-\bar{Y}, \quad \widetilde{Z} \leftrightarrow(-i,-2,1) \leftrightarrow-\widetilde{Y}, \\
& \left\{L_{\alpha \beta}, L_{\dot{\alpha} \dot{\beta}}, B_{I J}, J_{i j}, A, B, D\right\} \leftrightarrow(0,0,0) .
\end{aligned}
$$

(Анти)коммутаторы. Очевидно, что все ненулевые (анти)коммутируюшие соотношения между 24 генераторами, выписанные в явном виде, потребуют слишком много места. Поэтому мы выбрали такую параметризацию, при которой благодаря симметрии меж ду сектором

$$
\left(P_{\alpha \dot{\alpha}}, p_{\dot{\alpha} i}, q_{\alpha i}, W_{\alpha \dot{\alpha}}, \bar{\omega}_{\dot{\alpha} i}, Z_{\alpha i}, Z, \bar{Z}, \widetilde{Z}, Q_{\alpha I}, Q_{\dot{\alpha} I}, Q_{i I}\right)
$$

и обратным к нему по отношению к $(A, B, D)$-градуировкам сектором

$$
\left(K_{\alpha \dot{\alpha}}, k_{\alpha i}, s_{\alpha i}, \bar{W}_{\alpha \dot{\alpha}}, \omega_{\dot{\alpha} i}, Y_{\alpha i}, Y, \bar{Y}, \tilde{Y}, R_{\alpha I}, R_{\dot{\alpha} I}, R_{i I}\right)
$$

не было необходимости выписывать все соотношения. Разделим все (анти)коммутаторы на три класса: А, В, С. Симметричные соотношения из класса А получаются прямой заменой генераторов одного сектора соответствуюшими генераторами другого; соотношения класса В - заменой с обратным знаком; а соотношения из класса С представляют собой (анти)коммутаторы между обратными генераторами. В качестве примера приведем образец получения симметричных соотношений классов А и В:

$$
\begin{aligned}
& \mathrm{A}: \quad 2 \varepsilon_{\alpha \beta} Q_{\dot{\alpha} I}=\left[P_{\alpha \dot{\alpha}}, R_{\beta I}\right] \rightarrow 2 \varepsilon_{\alpha \beta} R_{\dot{\alpha} I}=\left[K_{\alpha \dot{\alpha}}, Q_{\beta J}\right] \\
& \mathrm{B}: \quad-\varepsilon_{i j} W_{\alpha \dot{\alpha}}=\left[q_{\alpha i}, \omega_{\dot{\alpha} j}\right] \rightarrow \varepsilon_{i j} \bar{W}_{\alpha \dot{\alpha}}=\left[s_{\alpha i}, \bar{\omega}_{\dot{\alpha} j}\right] .
\end{aligned}
$$


Все коммутаторы, в которых не участвуют генераторы $Q$ и $R$, а также все антикоммутаторы принадлежат классу В.

В построенной алгебре мы имеем 18 коммутирующих твисторных, векторных и скалярных генераторов и соответственно 18 “порождающих" их нетривиальных антикоммутаторов:

$$
\begin{aligned}
\left\{Q_{\alpha I}, Q_{\dot{\alpha} J}\right\}_{+}=-\varepsilon_{I J} P_{\alpha \dot{\alpha}}, & \left\{Q_{\alpha I}, R_{\dot{\alpha} J}\right\}_{+}=\varepsilon_{I J} W_{\alpha \dot{\alpha}}, & \left\{Q_{\alpha I}, Q_{\beta J}\right\}_{+}=2 \varepsilon_{\alpha \beta} \varepsilon_{I J} Z, \\
\left\{Q_{\dot{\alpha} I}, Q_{i J}\right\}_{+}=-\varepsilon_{I J} p_{\dot{\alpha} i}, & \left\{Q_{\dot{\alpha} I}, R_{i J}\right\}_{+}=\varepsilon_{I J} \bar{\omega}_{\dot{\alpha} i}, & \left\{Q_{\dot{\alpha} I}, Q_{\dot{\beta} J}\right\}_{+}=\varepsilon_{\dot{\alpha} \dot{\beta}} \varepsilon_{I J} \bar{Z}, \\
\left\{Q_{\alpha I}, Q_{i J}\right\}_{+}=\varepsilon_{I J} Z_{\alpha i}, & \left\{Q_{\alpha I}, R_{i J}\right\}_{+}=\varepsilon_{I J} q_{\alpha i}, & \left\{Q_{i I}, Q_{j J}\right\}=\varepsilon_{i j} \varepsilon_{I J} \widetilde{Z} .
\end{aligned}
$$

Сушествуют 60 нетривиальных коммутаторов между этими 18 генераторами:

$$
\begin{aligned}
& -\varepsilon_{i j} P_{\alpha \dot{\alpha}}=\left[q_{\alpha i}, p_{\dot{\alpha} j}\right]=\left[z_{\alpha i}, \bar{\omega}_{\dot{\alpha} j}\right], \quad-\varepsilon_{i j} W_{\alpha \dot{\alpha}}=\left[q_{\alpha i}, \omega_{\dot{\alpha} j}\right]=-\left[z_{\alpha i}, k_{\dot{\alpha} j}\right], \\
& -2 \varepsilon_{\alpha \beta} p_{\dot{\alpha} i}=\left[P_{\alpha \dot{\alpha}}, s_{\beta i}\right]=\left[\bar{W}_{\alpha \dot{\alpha}}, Z_{\beta i}\right], \quad-2 \varepsilon_{\alpha \beta} \bar{\omega}_{\dot{\alpha} i}=\left[P_{\alpha \dot{\alpha}}, Y_{\beta i}\right]=-\left[\bar{W}_{\alpha \dot{\alpha}}, q_{\beta i}\right] \text {, } \\
& -2 \varepsilon_{\dot{\alpha} \dot{\beta}} Z_{\alpha i}=\left[P_{\alpha \dot{\alpha}}, \omega_{\dot{\beta} i}\right]=-\left[W_{\alpha \dot{\alpha}}, p_{\dot{\beta} i}\right], \quad-2 \varepsilon_{\dot{\alpha} \dot{\beta}} q_{\alpha i}=\left[P_{\alpha \dot{\alpha}}, k_{\dot{\beta} i}\right]=\left[W_{\alpha \dot{\alpha}}, \omega_{\dot{\beta} i}\right], \\
& {\left[P_{\alpha \dot{\alpha}}, W_{\beta \dot{\beta}}\right]=4 \varepsilon_{\alpha \beta} \varepsilon_{\dot{\alpha} \dot{\beta}} Z, \quad\left[P_{\alpha \alpha}, \bar{W}_{\beta \dot{\beta}}\right]=-4 \varepsilon_{\alpha \beta} \varepsilon_{\dot{\alpha} \dot{\beta}} \bar{Z}, \quad\left[s_{\alpha i}, Z_{\beta j}\right]=2 \varepsilon_{\alpha \beta} \varepsilon_{i j} \widetilde{Z},} \\
& {\left[q_{\alpha i}, Z_{\beta j}\right]=-2 \varepsilon_{\alpha \beta} \varepsilon_{i j} Z, \quad\left[p_{\dot{\alpha} i}, \bar{\omega}_{\dot{\beta} j}\right]=-2 \varepsilon_{\dot{\alpha} \dot{\beta}} \varepsilon_{i j} \bar{Z}, \quad\left[p_{\dot{\alpha} i}, \omega_{\dot{\beta} j}\right]=2 \varepsilon_{\dot{\alpha} \dot{\beta}} \varepsilon_{i j} \widetilde{Z},}
\end{aligned}
$$

а также коммутаторы, определяющие действия центральных зарядов:

$$
\begin{aligned}
{\left[Z, \bar{W}_{\alpha \dot{\alpha}}\right] } & =-\left[\bar{Z}, W_{\alpha \dot{\alpha}}\right]=P_{\alpha \dot{\alpha}}, & -\left[\bar{Z}, \omega_{\dot{\alpha} i}\right] & =-\left[\widetilde{Z}, \bar{\omega}_{\dot{\alpha} i}\right]=p_{\dot{\alpha} i}, \\
{\left[Z, s_{\alpha i}\right] } & =-\left[\widetilde{Z}, q_{\alpha i}\right]=Z_{\alpha i}, & -\left[Z, K_{\alpha \dot{\alpha}}\right] & =-\left[\widetilde{Y}, P_{\alpha \dot{\alpha}}\right]=W_{\alpha \dot{\alpha}}, \\
{\left[\bar{Z}, k_{\alpha i}\right] } & =-\left[\widetilde{Y}, p_{\dot{\alpha} i}\right]=\bar{\omega}_{\dot{\alpha} i}, & {\left[Z, Y_{\alpha i}\right] } & =\left[\widetilde{Y}, Z_{\alpha i}\right]=q_{\alpha i} .
\end{aligned}
$$

Коммутаторы генераторов центральных зарядов с суперсдвигами и суперконформными бустами (набором $\{Q, R\})$, напротив, принадлежат классу А:

$$
\left[Z, R_{\alpha I}\right]=-Q_{\alpha I}, \quad\left[\bar{Z}, R_{\dot{\alpha} I}\right]=Q_{\dot{\alpha} I}, \quad\left[\widetilde{Z}, R_{i I}\right]=-Q_{i I}
$$

а нетривиальные соотношения между набором $\{Q, R\}$ и безразмерным (размерным) относительно дилатации набором $\left\{W_{\alpha \dot{\alpha}}, \bar{W}_{\alpha \dot{\alpha}}, \omega_{\alpha i}, \bar{\omega}_{\dot{\alpha} i}, q_{\alpha i}, s_{\alpha i}\right\}$ (соответственно $\left\{P_{\alpha \dot{\alpha}}\right.$, $\left.\left.K_{\alpha \dot{\alpha}}, p_{\dot{\alpha} i}, k_{\dot{\alpha} i}, Z_{\dot{\alpha} i}, Y_{\dot{\alpha} i}\right\}\right)$ принадлежат к классу В (соответственно А):

$$
\begin{aligned}
& \text { B: }\left[q_{\alpha i}, Q_{j I}\right]=\varepsilon_{i j} Q_{\alpha I}, \quad\left[\bar{W}_{\alpha \dot{\alpha}}, Q_{\beta I}\right]=2 \varepsilon_{\alpha \beta} Q_{\dot{\alpha} I}, \quad\left[s_{\alpha i}, Q_{\beta I}\right]=2 \varepsilon_{\alpha \beta} Q_{i I}, \\
& {\left[W_{\alpha \dot{\alpha}}, Q_{\dot{\beta} I}\right]=-2 \varepsilon_{\dot{\alpha} \dot{\beta}} Q_{\alpha I}, \quad\left[\bar{\omega}_{\dot{\alpha} i}, Q_{j I}\right]=\varepsilon_{i j} Q_{\dot{\alpha} I}, \quad\left[\omega_{\dot{\alpha} i}, Q_{\dot{\beta} I}\right]=2 \varepsilon_{\dot{\alpha} \dot{\beta}} Q_{i I} ;} \\
& \mathrm{A}:\left[Z_{\alpha i}, R_{j I}\right]=-\varepsilon_{i j} Q_{\alpha I}, \quad\left[P_{\alpha \dot{\alpha}}, R_{\beta I}\right]=2 \varepsilon_{\alpha \beta} Q_{\dot{\alpha} I}, \quad\left[Z_{\alpha i}, R_{\beta I}\right]=-2 \varepsilon_{\alpha \beta} Q_{i I} \text {, } \\
& {\left[P_{\alpha \dot{\alpha}}, R_{\dot{\beta} I}\right]=-2 \varepsilon_{\dot{\alpha} \dot{\beta}} Q_{\alpha I}, \quad\left[p_{\dot{\alpha} i}, R_{j I}\right]=\varepsilon_{i j} Q_{\dot{\alpha} I}, \quad\left[p_{\dot{\alpha} i}, R_{\dot{\beta} I}\right]=2 \varepsilon_{\dot{\alpha} \dot{\beta}} Q_{i I} .}
\end{aligned}
$$

Перечень (анти)коммутаторов, определяюших полученную алгебру, завершают соотношения группы C, записанные для компактности по два в строке:

$$
\left\{R_{\alpha(\dot{\alpha}) I}, Q_{\beta(\dot{\beta}) J}\right\}=2 \varepsilon_{\alpha \beta(\dot{\alpha} \dot{\beta})} B_{I J}-\varepsilon_{I J} L_{\alpha \beta(\dot{\alpha} \dot{\beta})}-i \varepsilon_{\alpha \beta(\dot{\alpha} \dot{\beta})} \varepsilon_{I J}[i B-D+2(0) A],
$$




$$
\begin{gathered}
\left\{R_{i I}, Q_{j J}\right\}=2 \varepsilon_{i j} B_{I J}-\varepsilon_{I J} L_{i j}-\varepsilon_{i j} \varepsilon_{I J}\left[\frac{i}{2} A\right] \\
{\left[P(W)_{\dot{\alpha} \alpha}, K(\bar{W})_{\dot{\beta} \beta}\right]=2 \varepsilon_{\dot{\alpha} \dot{\beta}} L_{\alpha \beta}+2 \varepsilon_{\alpha \beta} L_{\dot{\alpha} \dot{\beta}}+2 i \varepsilon_{\dot{\alpha} \dot{\beta}} \varepsilon_{\alpha \beta}[-0(i) B+2(0) D-A],} \\
{\left[p(\bar{\omega})_{\dot{\alpha} i}, k(\omega)_{\dot{\beta} j}\right]=-2 \varepsilon_{\dot{\alpha} \dot{\beta}} J_{i j}-\varepsilon_{i j} L_{\dot{\alpha} \dot{\beta}} \mp i \varepsilon_{\dot{\alpha} \dot{\beta}} \varepsilon_{i j}[i B+D \pm A]} \\
{\left[q(Z)_{\alpha i}, s(Y)_{\beta j}\right]=-2 \varepsilon_{\alpha \beta} J_{i j}-\varepsilon_{i j} L_{\alpha \beta}-i \varepsilon_{\alpha \beta} \varepsilon_{i j}[i B \mp D+2(-1) A],} \\
{[Z, Y]=B+i(D-A), \quad[\bar{Z}, \bar{Y}]=i D-B, \quad[\widetilde{Z}, \widetilde{Y}]=i A .}
\end{gathered}
$$

Генератор $b_{I A}$. Наконец, последний элемент в нашей конструкции состоит в допустимости применения той же процедуры к изотопической группе $S U(2)$ :

$$
\begin{gathered}
{\left[b_{I A}, b_{J B}\right]=-\varepsilon_{I J} B_{A B}-\varepsilon_{A B} B_{I J}, \quad\left[b_{I A}, Q \cdot J\right]=\varepsilon_{I J} Q \cdot A, \quad\left[b_{I A}, Q \cdot B\right]=\varepsilon_{A B} Q \cdot I} \\
\left\{R_{\alpha(\dot{\alpha}) A}, Q_{\beta(\dot{\beta}) I}\right\}=\left\{R_{\alpha(\dot{\alpha}) I}, Q_{(\beta) \dot{\beta} A}\right\}=2 \varepsilon_{\alpha \beta(\dot{\alpha} \dot{\beta})} b_{I A}, \\
\left\{R_{i A}, Q_{j I}\right\}=\left\{R_{i I}, Q_{j A}\right\}=\varepsilon_{i j} b_{I A} .
\end{gathered}
$$

Здесь твисторный генератор $b_{I A}$ переводит изотопические индексы $\{I, J, K, \ldots\}$ "касательного" пространства в "мировые" индексы $\{A, B, C, \ldots\}$, которые также являются индексами спиновой $S U(2)$-симметрии; генератор $B_{A B}$ в этом смысле полностью аналогичен $B_{I J}$.

Таким образом, построенная алгебра решает задачу спинорной параметризации супералгебры, описываюшей $N=2$ суперпространство с нетривиальными центральными зарядами.

\section{4. ПЕРСПЕКТИВЫ}

Мы считаем, что развитие представленной конструкции по ряду направлений является весьма перспективным. Замечено, в частности, что набор существенных координат сужается за счет привлечения безразмерных гармоник, параметризующих генераторы $b_{I A}, \bar{\omega}_{\dot{\alpha} i}, q_{\alpha i}$, до гармоник $\zeta^{\alpha i}, x^{\dot{\alpha} i}, \theta^{i A}$, параметризуюших, в свою очередь, генераторы $\widehat{Z}_{\alpha i}, \hat{p}_{\dot{\alpha} i}, \widehat{Q}_{i A}$. В случае $N=1$ число грассмановых размерностей сводится минимум к трем: $\theta_{\dot{\alpha}}, \theta_{i=1}$, что указывает на своеобразное проявление $k$-суперсимметрии Зигеля [1]. Обратим внимание на то, что стандартные грассмановы координаты образуются за счет домножения $\theta_{i A}$ - скаляра по Лоренцу - на соответствуюшую гармонику.

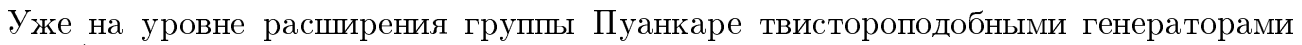
$(\hat{q}, \hat{s} ; \hat{p}, \hat{k})$ можно показать, что постулируемое Пенроузом основное твисторное уравнение [4] возникает как ограничение касательного расслоения реперов этого группового многообразия (уравнения Пфаффа).

Можно выбрать сечения (нормальные координаты) на фактор-группе следуюшим образом:

$$
e^{\left(\frac{2 i}{5} a \widetilde{A}\right)} e^{\left(\frac{i}{2} x^{\dot{\alpha} \alpha} \hat{P}_{\dot{\alpha} \alpha}+i \omega^{\alpha} \hat{q}_{\alpha 1}\right)} e^{\left(i \pi^{\dot{\alpha}} \hat{k}_{\dot{\alpha} 1}\right)} e^{\left(\frac{i}{2} \bar{\omega}^{\dot{\alpha}} \hat{p}_{\dot{\alpha} 2}+i \pi^{\alpha} \hat{s}_{\alpha 2}\right)},
$$

где $\widetilde{A}=\widehat{A}+2 \hat{J}_{12}$ задает базис векторных полей. На пересечении $\alpha$ - и $\beta$-плоскостей имеется набор соотношений, согласованных с квантово-механической интерпретацией твисторных переменных,

$$
\omega^{\alpha} \pi_{\beta}-\omega_{\beta} \omega^{\alpha}=\delta_{\beta}^{\alpha}, \quad \omega^{\alpha} \pi_{\alpha}=1, \quad \omega^{\dot{\alpha}} \pi_{\dot{\alpha}}=1 .
$$


При этом координаты вешественного пространства Минковского могут быть откалиброваны полностью: $x= \pm i \omega \bar{\omega}$. Решения уравнения связи

$$
D_{a} \Phi=\left(\partial_{a}-i \omega \partial_{\omega}+i \bar{\omega} \partial_{\bar{\omega}}\right) \Phi=0
$$

с естественным условием цикличности по переменной $a$ представляют собой поля левой или правой киральности:

$$
\Phi=e^{i n a} \omega^{\alpha(\dot{\alpha})} \cdots \psi_{\alpha(\dot{\alpha})}, \quad n=0, \pm 1, \pm 2 \ldots
$$

Вторая связь

$$
D_{\alpha \dot{\alpha}} \Phi=\left(\partial_{\alpha \dot{\alpha}}-\pi_{\dot{\alpha}} \partial_{\alpha}-\pi_{\alpha} \partial_{\dot{\alpha}}\right) \Phi=0
$$

является свободным уравнением движения безмассовых полей. Существенно, что на пространстве $\omega, \bar{\omega}$ “живут” поля либо только левой, либо только правой киральности.

В перспективе представляется интересным исследовать структуры, возникающие при гармонизации $N=2$ суперпространства, конструированием гармоник из параметризующих генератор $J_{i j}$ “углов" $\phi^{i j}$. Предварительные исследования показывают, что на этом пути нас ждут красивые геометрические объекты и прямая связь с гармониками Огиевецкого.

Так как набор $\left(W_{\alpha \dot{\alpha}}, \bar{W}_{\alpha \dot{\alpha}}, L_{\alpha \beta}, L_{\dot{\alpha} \dot{\beta}}, B\right)$ представляет собой подгруппу, изоморфную $S O(1,5)$, естественно ожидать, что голдстоуновские моды $a^{\alpha \dot{\alpha}}, \bar{a}^{\alpha \dot{\alpha}}$ нарушенной лоренш-подобной $\left(W_{\alpha \dot{\alpha}}, \bar{W}_{\alpha \dot{\alpha}}\right)$ симметрии связаны с мультиплетом гравифотона. При этом компоненты кручения для 1 -фрормы $E_{(Z)}=d x^{\mu} a_{\mu}+\cdots$ :

$$
T_{(Z)}=i E^{\alpha I} \wedge E_{\alpha I}+E^{\mu} \wedge \Omega_{\mu}
$$

где $\Omega_{\mu}=d a_{\mu}+\cdots$, являются соответствуюшими напряженностями. Таким образом, возникает надежда последовательно описать различные варианты супергруппы Пуанкаре для всех $N$ и $D$ как различные фазы спонтанного нарушения максимального расширения суперконформной симметрии.

В радикальной модификации струнной картины посредством замены римановых поверхностей и функций обобшенными твисторными пространствами и элементами голоморфного пучка когомологий [7] ожидаются такие же привлекательные свойства точной интегрируемости, как и в $2 D$-конформных теориях поля.

Предлагаемый спектр алгебраических конструкций с естественной поляризацией дает единообразные методы исследования голоморфной классификации главных $G$-расслоений и их деформаций, играющих, как представляется, важную роль в решении нелинейных задач квантовой теории поля.

Таким образом, начиная со спинорной параметризации фактора $S O(1,5) / S O(1,3)$ мы пришли, подчиняясь условиям непротиворечивости, к алгебраическим структурам, связывающим в единое групповое многообразие разнообразные элементы твисторных теорий. Мы надеемся, что исследование возникающих из нарушения симметрий геометрических структур позволит осознать источник компенсаторов и препотенциальных формулировок, а также классификацию связей расширенных супергрупп, где суперсимметрия и твисторы нуждаются друг в друге. Важно подчеркнуть, что подобные расширения супералгебр возникают и для других значений $N$ и $D$. Например, при $D=10$ $\operatorname{dim} Z=126, \operatorname{dim} W=210$ или при $D=11 \operatorname{dim} Z=517, \operatorname{dim} W=969$ [8].

Мы благодарны В. В. Серебрякову за интерес к работе и ее обсуждение. Работа выполнена при финансовой поддержке РФФИ (код проекта 93-02-15706). 


\section{Список литературы}

[1] W. Ziegel. Phys. Lett. B. 1983. V. 128. P. 397.

[2] D. P. Sorokin, V. I. Tkach, D. V. Volkov. Mod. Phys. Lett. 1989. V. A4. P. 901.

[3] В. И. Огиевецкий, Л. Мезинческу. УФН. 1975. Т. 117. Вып. 4.

[4] R. Penrose, M. A. H. MacCallum. Phys. Rep. 1972. № 6. P. 241.

[5] A. Galperin, E. Ivanov, S. Kalitzin, V. Ogievetsky, E. Sokatchev. Class. Quantum Grav. 1985. № 2. P. 601.

[6] J. C. Taylor. J. Phys. A. 1981. № 15. P. 867; Phys. Lett. B. 1981. V. 105. P. 434.

[7] A. P. Hodegs, R. Penrose, M. A. Singer. Phys. Lett. B. 1989. V. 216. P. 48.

[8] Н. Г. Плетнев, В. В. Серебряков. ТМФ. 1987. Т. 70. С. 255; N. G. Pletnev. J. Mod. Phys. A. 1991. № 6. P. 4667.

Поступила в редакцию 10.I.1996 г.

\section{N. G. Pletnev, D. V. Serebryakova \\ SUPERALGEBRA OF CONFORMAL DEFORMATIONS FOR TWISTOR-LIKE SUPERSPACES}

A twistor-like extension of $N=2$ conformal superspace (SSp) with nontrivial central charges is presented. Parameters of new (anti)commutative generators carring an index of the spin $S U(2)$ symmetry are used to construct massless harmonics reducing thereby a number of essential coordinates. In this approach the usual vector coordinates become complex while the Grassmann ones are reduced to a single variable being scalar under the Lorentz transformation. A choice of an appropriate analytical subspace on an initial SSp is rather difficult, but our closed algebra gives the possibility to reduce covariantly the theory from the initial SSp to a SSp of essentially smaller Grassmann dimension. The definition of coordinates and flat space in this structure allows one to illustrate how the twistor equation of Penrose and GIKOS-harmonics arise. 